\title{
Editorial
}

\section{What does the future hold for BNP in cardiology?}

The identification of natriuretic peptides as key regulators of natriuresis and vasodilatation, and the appreciation that their secretion is under the control of cardiac haemodynamic and neurohumoral factors, have boosted a wide interest among scientists and cardiologists in the last 10 years. Brain natriuretic peptide (BNP, 32 amino acids) is secreted into the circulation mainly by left ventricular (and atrial) myocytes in conditions of overload or wall stress (dilatation and/or abnormal filling pressure). Through its binding to specific membrane receptors (NPR-A), the hormone generates the second messenger cGMP and stimulates the nitric oxide system. BNP may be envisioned as a "good" hormone that counteracts the effects of the sympathetic and renin-angiotensin-aldosterone systems by favouring peripheral vasodilatation, renal excretion of water and sodium, and by inhibiting abnormal vascular cell growth.

The authors of this commentary share most of the views expressed in two recent editorials. ${ }^{12}$ Plasma BNP concentration rises with the severity of cardiac disease. An early report showed how the plasma BNP-like immunoreactive level was increased in patients with heart failure as a function of New York Heart Association (NYHA) functional class. $^{3}$ Since then, virtually all clinical, functional, and instrumental indexes of the severity of heart failure have been found to be related to circulating BNP concentration. The validity of BNP as a reliable and powerful indicator of left ventricular (LV) dysfunction has been recently confirmed in the largest biohumoral database from a randomised clinical trial, Val-HeFT (valsartan in heart failure trial). ${ }^{4} \mathrm{~A}$ biohumoral profile at study entry has been performed in most $(85 \%)$ of the 5010 patients with heart failure enrolled. A preliminary analysis of this database revealed that BNP was the best indicator of the severity of heart failure, compared to renin activity, aldosterone, noradrenaline (norepinephrine), and endothelin, also assayed at study entry. For example, the ratio between circulating factors measured in the population of patients with an LV internal diastolic diameter greater than the median value $\left(>3.57 \mathrm{~cm} / \mathrm{m}^{2}\right)$, and in those with a diameter less than the median, was 1.99 for BNP, compared to 1.21 for endothelin-1, 1.13 for noradrenaline, 1.08 for renin activity, and 1.02 for aldosterone. ${ }^{5}$ Using the same approach, $\mathrm{BNP}$ also appeared to be the best indicator of LV dysfunction; the ratio of BNP concentrations among patients with an LV ejection fraction (LVEF) less than the median value $(27 \%)$ and patients with a more preserved function (LVEF $27 \%$ ) was 1.62 for BNP, and 1.52, 1.21, 1.14, and 1.12 for renin activity, endothelin-1, aldosterone, and noradrenaline, respectively. Finally, BNP was the best indicator of a worse NYHA functional class.

\section{BNP following myocardial infarction}

BNP has emerged more recently as a prognostic indicator early (three days) after myocardial infarction. ${ }^{67}$ In this issue of Heart, Crilley and Farrer report that patients with higher plasma BNP concentrations measured within the first week after a first myocardial infarction experience adverse left ventricular remodelling as assessed by echocardiography. ${ }^{8}$ In this study, BNP was a good predictor of one year cardiac mortality.

The chemical stability of the molecule, ${ }^{9}$ and the availability of sensitive, reliable, relatively simple, and reproducible assays, have favoured a diffusion of BNP measurement out of research towards clinical chemistry laboratories of general hospitals. Pilot UK experiences in the general population ${ }^{10}$ have been transferred to other contexts: in a nationwide network of clinical centres (Italian Network on Congestive Heart Failure, IN-CHF) BNP immunoradiometric assay (Shionogi) was performed in 20 different clinical chemistry laboratories without previous experience of the test. Results were encouraging for a generalisation of this test in ambulatory patients with congestive heart failure. ${ }^{11}$ In summary, BNP (and also N-terminal proBNP and N-terminal proANP) is probably the best biohumoral indicator of LV dysfunction and prognosis in heart failure and after myocardial infarction. A word of caution-little is known about the large population of elderly patients with symptoms of heart failure but preserved left ventricular systolic function. We do not know whether or not measuring BNP will help in assessing this group of patients.

\section{BNP in the clinical setting}

How can BNP be used in everyday clinical cardiology? Two promising directions are currently undergoing evaluation:

- BNP may serve as a measure of the therapeutic effect of agents which do not have simple, quantitative indexes of efficacy that can reflect drug response in real time (that is, angiotensin converting enzyme (ACE) inhibitors). As a further step in that direction, BNP (or related peptides) have been proposed as a guide to optimise individual treatment of chronic heart failure ${ }^{12} 13$

- BNP may help in the diagnosis of heart failure in primary care, in particular in patients with other disorders such as chronic obstructive pulmonary disease presenting with dyspnoea or fatigue, or both. ${ }^{14}$ More importantly, the knowledge of plasma BNP concentration, alone or in combination with other simple diagnostic tools, could help the general practitioner to decide whether to refer a patient with suspected heart failure for cardiac imaging, still the most effective way of establishing a positive diagnosis.

Using the criteria of abnormal ECG alone reduces the need for echocardiography to be performed in patients with suspected heart failure, but can overlook as many as $10 \%$ of those with left ventricular systolic dysfunction. ${ }^{15}$ As a large scale screening strategy, it could be therefore proposed that patients in primary care with suspected heart failure, but a normal plasma BNP concentration (for example, $<76 \mathrm{pg} / \mathrm{ml}$ ) and normal ECG, would not be referred for cardiological consultancy and limited access echocardiographic examination.

It is expected that BNP measurement will be part of the algorithm for diagnosis of heart failure in the new European Society of Cardiology guidelines. What would then convince the cardiologist to include BNP (or related 
peptides) among routine biohumoral tests? We are still awaiting a new generation of simple yet more reliable methods. A bedside test (Triage BNP) has been approved by the US Food and Drug Agency as an aid in the diagnosis of congestive heart failure, and a multicentred clinical trial should be launched to assess its applicability to patient management. But, more importantly, the initial observation that BNP may be useful in the identification of patients with heart failure in primary care ${ }^{16}$ needs to be extended and confirmed in different and larger populations, and its impact on costs of heart failure patients' management assessed in different health care systems.

ROBERTO LATINI ALDO P MAGGIONI* SERGE MASSON

Department of Cardiovascular Research,

Istituto di Ricerche Farmacologiche "Mario Negri",

Milan, Italy

${ }^{\star} A N M C O$ Research Centre,

Florence, Italy

1 Cowie MR. BNP: soon to become a routine measure in the care of patients with heart failure? Heart 2000;83:617-8.

2 Hobbs R. Can heart failure be diagnosed in primary care ? Brain natriuretic peptide assays make it easier. BMF 2000;321:188-9.

3 Mukoyama $\mathrm{M}$, Nakao $\mathrm{K}$, Hosoda $\mathrm{K}$, et al. Brain natriuretic peptide as a Mukoyama $M$, Nakao $K$, Hosoda $\mathrm{K}$, et al. Brain natriuretic peptide as a
novel cardiac hormone in humans. Evidence for an exquisite dual natriuretic system, atrial natriuretic peptide and brain natriuretic peptide. $\mathcal{f}$ natriuretic system, atrial natriur
Clin Invest 1991;87:1402-12.
4 Cohn JN, Tognoni G, Glazer RD, et al. Rationale and design of the valsartan heart failure trial: a large multinational trial to assess the effects of heart failure trial: a large multinational trial to assess the effects of valsartan, an angiotensin-receptor blocker, on morbidity and

5 Latini R, Anand I, Masson S, et al. CHF severity and neurohormonal actiLatini R, Anand I, Masson S, et al. $\mathrm{CHF}$ severity and neurohormonal acti-
vation in a large international population with chronic heart failure enrolled in Val-HeFT [abstract]. 7 Am Coll Cardiol 2001;37:148A.

6 Darbar D, Davidson NC, Gillpesie N, et al. Diagnostic value of B-type natriuretic peptide concentrations in patients with acute myocardial infarction. Am $\mathcal{F}$ Cardiol 1996;78:284-7.

7 Omland T, Aakvaag A, Bonarjee VVS, et al. Plasma brain natriuretic peptide as an indicator of left ventricular systolic function and long-term survival after acute myocardial infarction. Comparison with plasma atrial natriuretic peptide and N-terminal proatrial natriuretic peptide. Circulation 1996;93:1963-9.

8 Crilley JG, Farrer M. Left ventricular remodelling and brain natriuretic peptide after first myocardial infarction. Heart 2001;86:638-42.

Gobinet-Georges A, Valli N, Filliatre $\mathrm{H}$, et al. Stability of brain natriuretic peptide (BNP) in human whole blood and plasma. Clin Chem Lab Med 2000;38:519-23.

10 McDonagh T, Robb SD, Murdoch DR, et al. Biochemical detection of left ventricular systolic dysfunction. Lancet 1998;351:9-13.

11 Masson S, Gorini M, Salio M, et al. Clinical correlates of elevated plasma natriuretic peptides and Big endothelin-1 in a population of ambulatory patients with heart failure. A substudy of the Italian network on congestive heart failure (IN-CHF) registry. Italian Heart fournal 2000;1:282-8.

12 Murdoch DR, McDonagh TA, Byrne J, et al. Titration of vasodilator therapy in chronic heart failure according to plasma brain natriuretic peptide concentration: randomized comparison of the hemodynamic and neuroendocrine effects of tailored versus empirical therapy. Am Heart $\mathcal{F}$ 1999;138: 1126-32.

13 Troughton RW, Frampton CM, Yandle TG, et al. Treatment of heart failure guided by plasma aminoterminal brain natriuretic peptide (N-BNP) concentrations. Lancet 2000;355:1126-30.

14 Davis M, Espiner E, Richards G, et al. Plasma brain natriuretic peptide in assessment of acute dyspnoea. Lancet 1994;343:440-4.

15 Houghton AR, Sparrow NJ, Toms E, et al. Should general practitioners use the electrocardiogram to select patients with suspected heart failure for the electrocardiogram to select patients with

16 Cowie MR, Struthers AD, Wood DA et al. Value of natriuretic peptides in assessment of patients with possible new heart failure in primary care. Lancet 1997;350:1347-51.

\section{IMAGES IN CARDIOLOGY}

\section{The "thumb and wrist sign" in Marfan syndrome}

A 16 year old male presented with dilatation of the ascending aorta $(41 \mathrm{~mm})$ and mitral valve prolapse. As shown below, the thumb protruded from the clenched fist and the wrist sign was also evident. Arachnodactily and loose joints account for the ability to position the fingers in this ways.

The patient also had other features of Marfan syndrome, including family history,

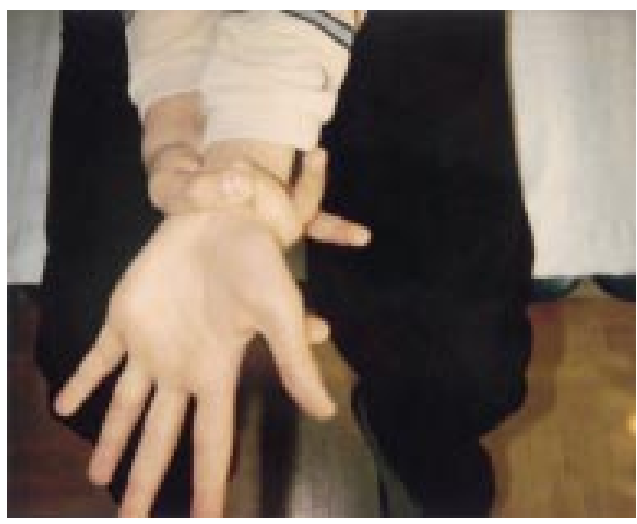

excessive height, span of the arms exceeding height, scoliosis of the spine, dilatation of the dural sac of the lumbar spine, a high arched palate, and ectopia lentis. Treatment with a $\beta$ blocker has been initiated.

GIUSEPPE COCCO gcocco@bluewin.ch

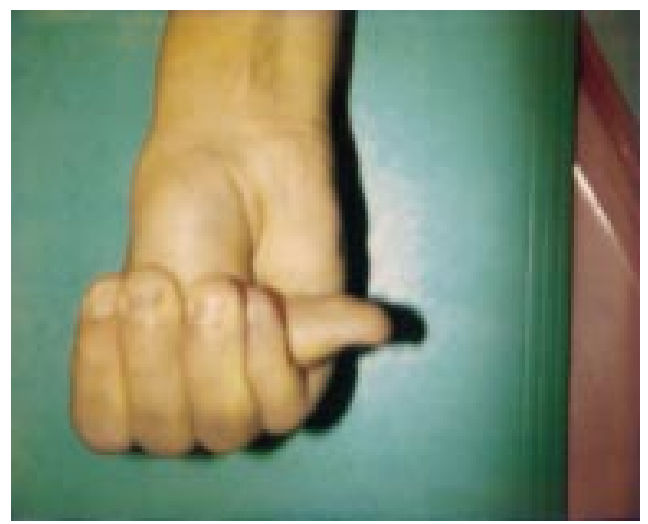

